Volume No. 1, Issue No. 3 (July-September), pp. 50-57

\title{
A Spatial 3 X 3 Average Filter for De-Noising in Digital Images with the Help of Median Filter \\ ${ }^{1}$ Alisha Kazmi, ${ }^{2}$ Samina Parveen, ${ }^{3}$ Sidra Sattar, ${ }^{4}$ Muhammad Nadeem Ali, ${ }^{5}$ Muhammad Adnan Khan
}

\begin{abstract}
Digital image processing includes many factors like Image enhancement, segmentation, object recognition, removal of noise and many more. Noise removal is the one of the hot area of image processing. Noise can be minimized but cannot removed completely. Scientist and researchers has established many filters, which can minimize the noise in the image and enhance its quality. There are many types of noise and many types of filter for the removal of noise. Many types of noise are used. To remove these types of noise, $3 \times 3$ average filter is used in this paper and its efficiency is measured. The simulations are performed on the MATLAB.
\end{abstract}

Key words - $3 \times 3$ Average Filter, Median Filter, Mean Filter, RSMF, VMF.

\section{INTRODUCTION}

A big challenge for researchers for Noise Removal in digital images to gain best performance. De-noising of image is an essential part of image reconstruction process. Many techniques were introduced to gain high ratio and effective result. Noise may be classified as substitutive noise, salt and pepper noise, additive noise like Gaussian noise and Speckle noise [1]. The important property of image de-noising system is that it should completely remove noise with preserving edges. De-noising the image before applying to different applications is an important factor.

7 Alisha Kazmi BSCS LGU, Lahore

2Samina Parveen BSCS LGU, Lahore,

3Sidra Sattar BSCS LGU, Lahore

${ }^{4}$ Muhammad Nadeem Ali Lecture LGU , Lahore ,

\section{FILTERS}

Filters are used to modify or enhance the image quality. For example, you can filter an image to emphasize particular features to reduce other features.

The low pass filter in digital image processing (DIP), can be represented as follows [1]

$$
\mathrm{D}(\boldsymbol{\omega}, \boldsymbol{k})=\frac{a}{a+\frac{k}{-i \omega}}
$$

Where $\mathrm{a}$ is the cutoff parameter, and the forward Fourier Kernel is defined as $\left(-i \omega t+i k_{x} x+i k_{z} z\right) \cdot{ }^{7}$

In order to determine a, let us suppose that the filter attenuates wave amplitudes by a factor of $\beta(\beta<1)$ at the boundary of

${ }^{5}$ Muhammad Adnan Khan Lecturer LGU , Lahore 
propagating region $k_{x} v / \omega=1$ that is

$$
\left|\boldsymbol{D}\left(\boldsymbol{\omega}, \boldsymbol{k}_{z}\right)\right|_{\frac{\boldsymbol{k}_{s} v}{\omega}=1}=\boldsymbol{\beta}
$$

The cutoff parameter a, of equation(i) can be found as,

$$
a=\frac{\omega_{0}}{v^{2} \sqrt{\left(\frac{1}{\beta}\right)^{2}-1}}
$$

Where $\omega_{0}$ is the dominant frequency, the low pass DIP-filtered output Q is related to the input $\mathrm{P}$ by

$$
\mathrm{Q}=\mathrm{DP}
$$

\subsection{Averaging Filter}

It is easy to understand average and mean filter, as these filters are simple. It does the function of smoothing of images (i.e lessening the variations of sharpness between two pixels) [2]. Average filter replaces every single pixel from the average of pixel in square shaped window enclosing these pixels. The greater window can discard noise in a better way but causing a blur or unfocused image. Exp. Is $\boldsymbol{g}(\boldsymbol{m}, \boldsymbol{n})=\sum_{l=-L}^{L} \sum_{k=-L}^{L} \boldsymbol{h}(\boldsymbol{k}, \boldsymbol{l}) \boldsymbol{s}(\boldsymbol{m}-$ $\boldsymbol{k}, \boldsymbol{n}-\boldsymbol{l})$

\section{2. $3 \times 3$ Average Filtering}

$3 \times 3$ average filter is in the form of a simple sliding-window, a dimensional filter that substitutes the center value with the average of all the pixel(dot) values included in the window. The window can be of any shape but mostly it is a square. It is the most famous and common low pass filter.

It upgrades the turbulent images, smooth the common differences and decreases the intensity. It exchanges the value of pixel by the representative vote of its $3 \times 3$ rectangular nearby region. It doesn't strain or filter and has to avoid the boundary rows and columns where just 6 or 4 (in the edges) neighbors prevail.

\section{IMAGE NOISE}

It is a normal variation (absent from the object image) of color and luster details in pictures. It is mostly a feature of automated sound. It can be formed by the feeler and circuitry system of a camera or a scanner.

\section{Types of Noises}

\section{Salt-and-Pepper noise}

A type of noise at times examined on images. It is seen as scarcely appearing pixels in white and black. Median or a morphological filter is an efficient method to reduce this kind of noise [3].

The PDF of (Bipolar) this known as Salt \& pepper noise

$$
\boldsymbol{p}(\boldsymbol{z})= \begin{cases}\boldsymbol{p}_{\boldsymbol{a}} & \text { for } \mathrm{z}=\mathrm{a} \\ \boldsymbol{p}_{\boldsymbol{b}} & \text { for } \mathrm{z}=\mathrm{b}\end{cases}
$$

Figure 1:- Salt and Pepper Noise 
Volume No. 1, Issue No. 3 (July-September), pp. 50-57

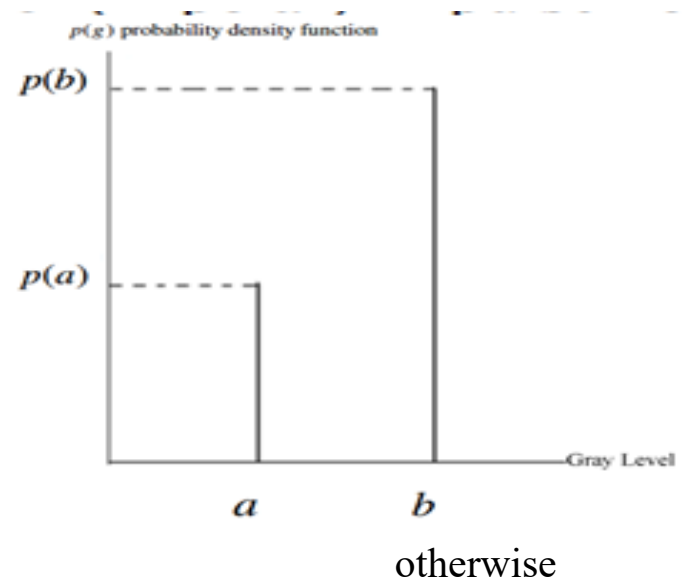

if $b>a$, gray-level $b$ appears as a light point in the image. Contrariwise, level seems like a dark point. If either $\mathrm{p}$ is zero, the impulse noise is called a Unipolar.

\subsubsection{Gaussian Noise}

$$
\mathrm{PG}(\mathrm{z})=\frac{1}{\sigma \sqrt{2 \pi}} e^{\frac{-(\mathrm{z}-\mu)^{2}}{2 \sigma^{2}}}
$$

In Digital Image Processing Gaussian Noise can be removed by Average Filter. To smooth the image from undesirable outcomes which blur the image [4].

Figure 4: - Speckle Noise Density Function

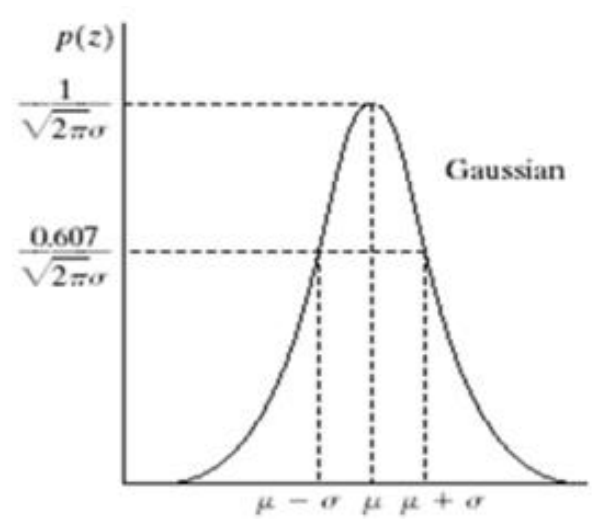

Figure2: - Gaussian Noise

\subsubsection{Local Variance}

variance is defined as how values are different from the average values.

$$
E\left[X^{2}\right]-(E[X])^{2}
$$

Instead of finding the variance for the whole matrix, variance is computed based on a small sliding a window.

\subsubsection{Poisson Noise}

This noise can be seen apparently, because of the statistical attributes of electromagnetic waves, for instance $\mathrm{x}$-rays $\&$ the gamma rays. The $\mathrm{x}$-rays and gamma rays discharged the number of photons per unit time. A source is used to insert these rays into patient's body in medical rays imaging systems. Those sources have unsystematic variations of photons. Outcome of assembled image has a spatial 
and secular
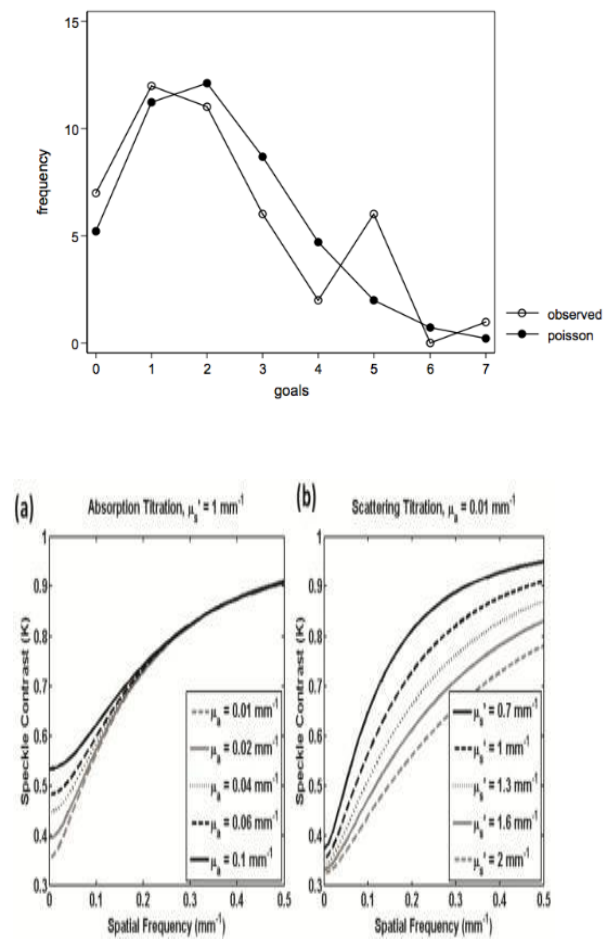

Figure 3: - Poisson Waveform

irregularity. This special noise is also known as quantum (photon) noise or shot noise. This noise accepts the Poisson distribution and it is given as

$$
\left.\boldsymbol{P}\left(\boldsymbol{f}_{(p i)}\right)=\boldsymbol{k}\right)=\frac{\lambda^{k} i^{e^{-\lambda}}}{k !}
$$

The magnitude of shot noise increases it, According to the square root of the supposed number of occurrence, such as the electric current or light force. But since the strength of the signal itself increases this more rapidly, the relative proportion of a shot noise reduces and a signal to noise ratio (considering only shot noise) increases anyway.

\subsubsection{Speckle Noise}

It is multiplicative or repeated noise. Their occurrence is seen in consistent imaging system such as laser, radar and acoustics etc. Speckle noise can lie alike in an image as Gaussian noise [5]. Its probability density function follows gamma distribution

$$
F(g)=\frac{g^{a-1} e^{\frac{-g}{a}}}{a-1 ! a^{a}}
$$




\section{SYSTEM MODEL}

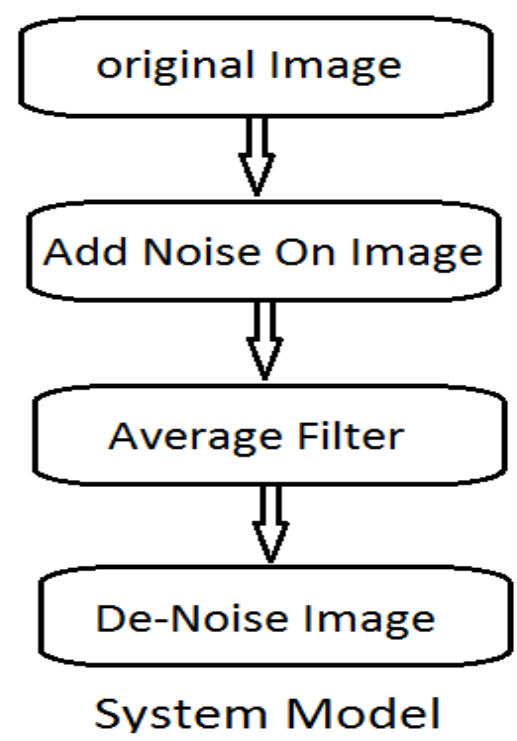

Figure 5: System Model

De-noising of noised image is simulated in this article. The first step is an original image is taken. The original is disturbed with noise. The various types of noises are added. Then an average filter of $3 \times 3$ is used to normalize or minimize the noise. After minimizing the various noises, all the results are compared. This system model which depict the working efficiency of average filter to various noises. The block diagram of system model is shown in figure:

\section{RESULTS: -}

In this noise, it is typically caused by faulty dots in the sensors, or timing errors in the digitization process. It is very clear, that $3 \times 3$ average filter is not removing salt and pepper noise effectively, and there are some dots that are showing the presence of noise. Although it is not upsetting the image quality, but it is not much fascinating for removal of salt and pepper noise. When this noise applied to an image, might be the minimal information is lost.

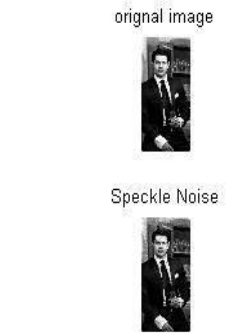

Average filter on Speckle noisy image

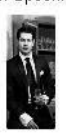

Figure 6: Average Filter on Speckle Noise 
It is a granular noise in any image which

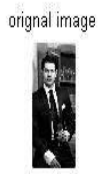

Poisson Noise

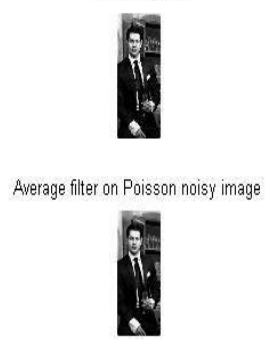

Figure 7: Average Filter on Poisson Noise

Makes the picture rough and affects its wavelength. The speckle can also represent some useful information when it is linked by laser speckle.

This Filter also works with Gaussian noise and continuous detailed information is better than the previous noises. According to result 3X3 Average filter doesn't work properly on local variance noise.

This result is better than the local variances' result. Because this result is much better and noiseless. orignal image

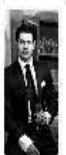

Local Variance Noise

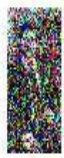

Average filter on Speckle noisy image

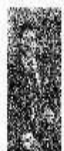

Figure 8: Average Filter on local variance noise.

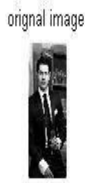

Gussian Noise

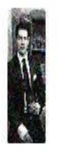

Average fitter on gaussian noisy image

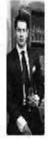

Figure 9: Average Filter on Gaussian Noise 
Volume No. 1, Issue No. 3 (July-September), pp. 50-57

\section{CONCLUSION}

In this research paper, various noise models Are implemented to minimize. Then an average filter of $3 \times 3$ is used. It is observed that average filter is working more efficiently for "salt $\boldsymbol{\&}$ pepper". To remove other noises, the filter type should be changed. 


\section{REFERENCES}

[1] R. R. a. A. Sharma, "Filters for image processing," IJARCSSE, vol. 5, no. 5, may, 2015.

[2] Hassh Prakteek Singh, Ayush Nigam, Amit Kumar Hautam, Aakanksha Bharadwaj and Neha Singh, "Average Filters," in ICACEA, 2014.

[3] D. A. G. S. E.Jebamalar Leavline, "Salt \& Peppar Noise, Reduction," IJSRSET, vol. 6, no. 05, 2013.

[4] D. Mr. Harikrishan, "Gaussian Noise Reduction," IJARCSSE, vol. 3, no. 9, 2013.

[5] M. H. a. A. Shah, "Reduction of speckle Noise," JIKRESCE. 